\title{
Broad-and Narrow-Beam Attenuation of 500- to 1,400- Kilovolt X-Rays in Lead and Concrete ${ }^{1}$
}

\author{
By Harold O. Wyckoff, Robert J. Kennedy, and William R. Bradford
}

Both narrow- and broad-beam attenuation curves have been obtained for 500-, 600-, 800-, 1,000-, and 1,400-kilovolt X-rays in lead and concrete. For the experimental condi. tions used, it is shown that an irradiated area 12 inches in diameter for lead absorbers and 37 inches in diameter for concrete satisfy the barrier conditions required for broad-beam attenuation curves.

\section{Introduction}

There have been numerous papers over the past 10 years dealing with $\mathrm{X}$-ray protection in the range from 500 to $2,000 \mathrm{kv}[1$ to 6$] .^{2}$ The data from these papers differ somewhat, because the conditions of the experiments were not always the same and therefore cannot be readily correlated. Part of this has been attributed to differences in the high-voltage wave form and inherent filtration for the several experimental arrangements used. Preliminary data $\left[\begin{array}{lll}1 & \text { to } & 6\end{array}\right]$ have indicated that the size of the irradiated area of the barrier may also be an important variable, but the magnitude and limits for this have not been explored quantitatively. The present report will deal with different sized beams of X-rays produced by direct-current potentials of $500,600,800,1,000$, and $1,400 \mathrm{kv}$ with a transmission target. Barriers of lead and concrete will be considered. Concrete has been generally accepted in the energy range above about $500 \mathrm{kv}$ where space is not important, because it is structurally self-supporting and relatively inexpensive. However, for applications requiring a minimum of thickness for a given protection, lead is still the most popular.

In the voltage range under consideration, X-rays are principally absorbed by the photoelectric and the Compton processes, since pair production is still relatively unimportant, even in lead for $1,400 \mathrm{kv}$. All of the photoelectric

1 This paper will also appear in Radiology.

2 Figures in brackets indicate the literature references at the end of this paper. absorption and that part of the energy transferred to the Compton electrons are considered to be truly absorbed, since the electrons so produced have small likelihood of producing another X-ray photon. The part of the energy given to the scattered photon leaves the site of the encounter in a different direction from that of the incident photon. The amount of this energy retained by the scattered photon decreases with increase of the angle between the scattered and incident photons. Thus, if the beam is of small cross section, the scattered photons leave the incident bundle and will not be measured. If, however, the beam is of considerable width the photons scattered from the sides of the beam may enter a measuring volume situated near the center of the beam. The apparent attenuation of the beam, measured by the reduction in dosage rate in the barrier, may thus be greatly influenced by the inclusion of this scattered radiation.

One may think of the ionization produced in the ionization chamber placed on the far side of a protective barrier as being due to two different sources of radiation. (a) Part of the radiation comes directly from the target of the X-ray tube. (The target is essentially a point source for all practical dimensions used in protective studies). (b) The remainder of the measured dosage may come from a distributed source composed of the entire irradiated volume of the protective barrier. Several factors serve to limit the volume, which acts as this secondary source. The length of the incident plus the scattered photon path in the barrier will be greater for oblique rays and, there- 
fore, obliquely incident photons have a greater probability of being absorbed. In addition, the angle between these incident and scattered photons must be larger and, therefore, the scattered photon energy will be smaller. The latter factor, depending upon the atomic number of the protective barrier, may also increase the probability of true absorption. In the X-ray range here considered, this factor is not important for concrete but serves to limit the practical dimensions of the virtual source when lead barriers are used.

Another factor producing differences between measurements made with and without scattering has recently been described [8]. It was shown there that air ionization chambers are more sensitive to photons of energies below $70 \mathrm{kv}$ than above. For increasing thickness of barrier, an equilibrium is soon established, so that as much of the low-energy radiation is absorbed as is created. As a result of this quality-dependent chamber sensitivity, one obtains an increase of dosage rate in protective barriers of low atomic number, such as concrete, where this low-energy radiation is not strongly absorbed. The attenuation curves are thus convex upward for small barrier thicknesses. On the other hand, for barriers of high atomic number such as lead, this effect is not observable, since the low-energy radiations are strongly absorbed in the barrier.

As can be easily seen from the above discussion, different experimental conditions may influence considerably the attenuation curve obtained. Two limiting conditions are thus defined for the work to be reported here. A narrow-beam attenuation curve shall be understood to be one where only a negligible amount of the scattered radiation from the barrier is measured in the ionization chamber. Practically, this condition amounts to having the irradiated area on the barrier subtend a small angle at the chamber. This condition may be verified experimentally by an inverse square check of the radiation received in the chamber. If the inverse square law is found to hold experimentally, with fixed position of the target and barrier but with variations of the target-to-chamber distance, then the contribution of scattered radiation from the barrier source is negligible.

Dosage readings - with a fixed position of the chamber, target, and barrier-are said to be for broad-beam conditions if, on increasing the irradiated area of the barrier, no increase in dosage rate is observed. This condition is, of course, only unique for that particular barrier-to-chamber distance. The above qualitative considerations indicate that the irradiated area required to fulfill broad-beam conditions will be larger for larger barrier-to-chamber distances. The requisite area may be reduced by shorter target-to-barrier distances and smaller chamber volumes.

Since some scattered radiation is measured by the chamber under broad-beam conditions but none is measured for narrow-beam conditions, the effective absorption coefficient must be different for the two conditions. The effective absorption coefficient is, however, proportional to the slope of the attenuation curve. Attenuation curves that include different amounts of scattered radiation in their determination should therefore have different slopes.

\section{Experimental Ārangement}

The high-voltage generator and tube have been previously described [7]. Figure 1 shows the experimental arrangement for beam collimation and for dosage measurement. The filtration inherent in the $\mathrm{X}$-ray tube was approximately $2.8 \mathrm{~mm}$ tungsten, plus $2.8 \mathrm{~mm}$ copper, plus $2.1 \mathrm{~mm}$ brass, plus $18.7 \mathrm{~mm}$ water for the center of the beam. The target protective housing consisted of a doublewalled steel tank filled with lead shot to a thickness of approximately 6 in., and a 6 -in. thick solid lead diaphragm. Three diaphragms of different apertures were used in the course of the experiments. They gave irradiated areas at the basement floor level whose diameters were approximately 13, 26, and $37 \mathrm{in}$., respectively. These dimensions were dictated on the lower end by the requirement of uniform irradiation of the chamber and on the upper end by a desire to minimize scattering from the pit walls.

Figure 2 shows a view of the pit in which the radiation measurements were made. The ionization chamber was connected to an evacuated cylinder housing an FP54 electrometer tube and a selection of resistors and could be moved by remote control both in azimuth and in elevation within the pit. (The chamber actually used had about one-twenty-fifth the volume of the one shown in fig. 2.) Remote switches controlled, and indicators defined, the position of the chamber in the pit, the resistor used, and the grid resistor boltage. The latter two figures, together with the volume 


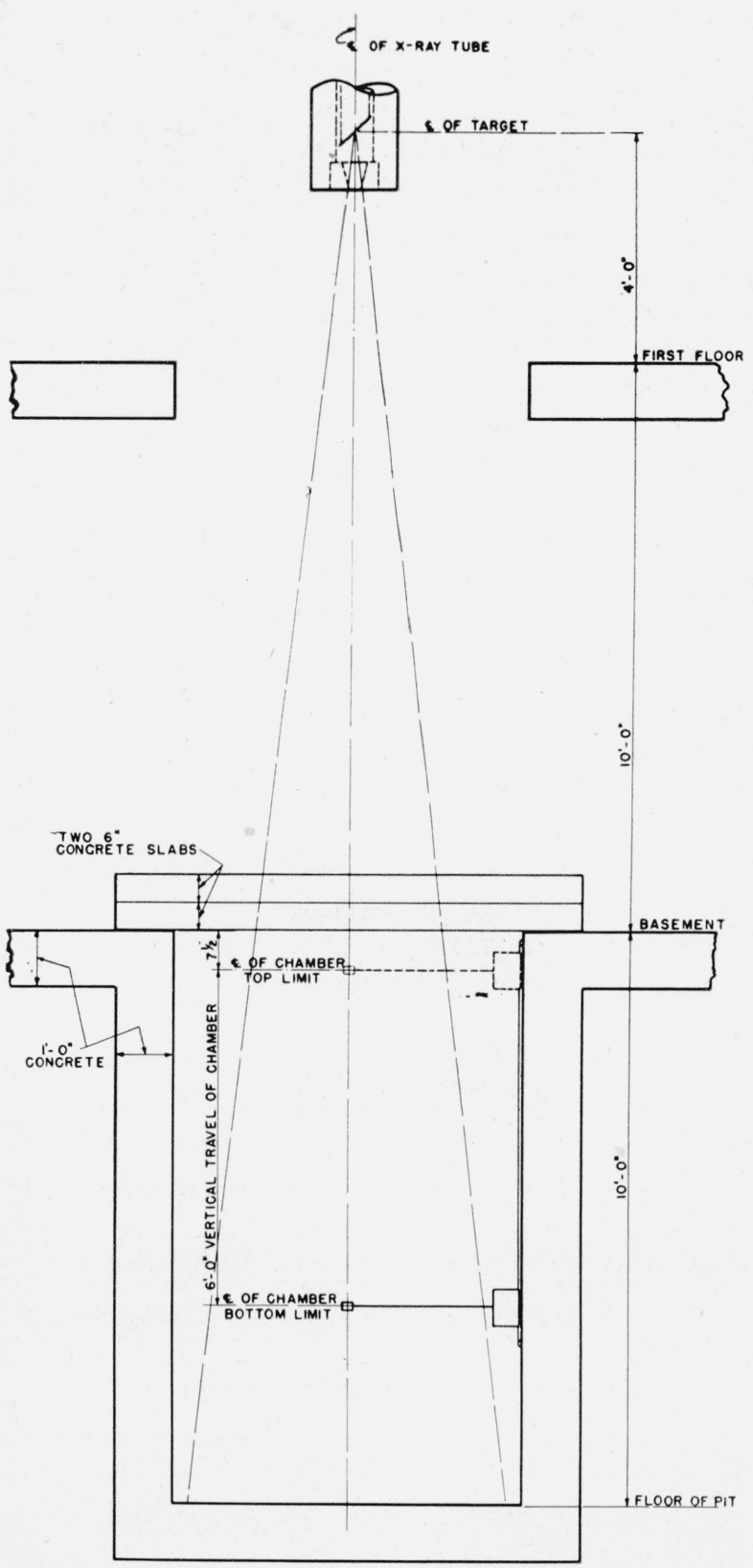

FIGURE 1. Experimental arrangement for collimating the beam and for dosage measuremeni.

of the chamber and the temperature and pressure of the air, served to determine the dosage rate. The chamber calibration determined from the value of the grid resistor, the grid voltage, and the mass of air in the chamber agrees to within 5 percent with that obtained experimentally with a standard radium source. By controlling the voltage and the current of the X-ray tube to within 0.1 percent, the $\mathrm{X}$-ray dosage was found to be con-

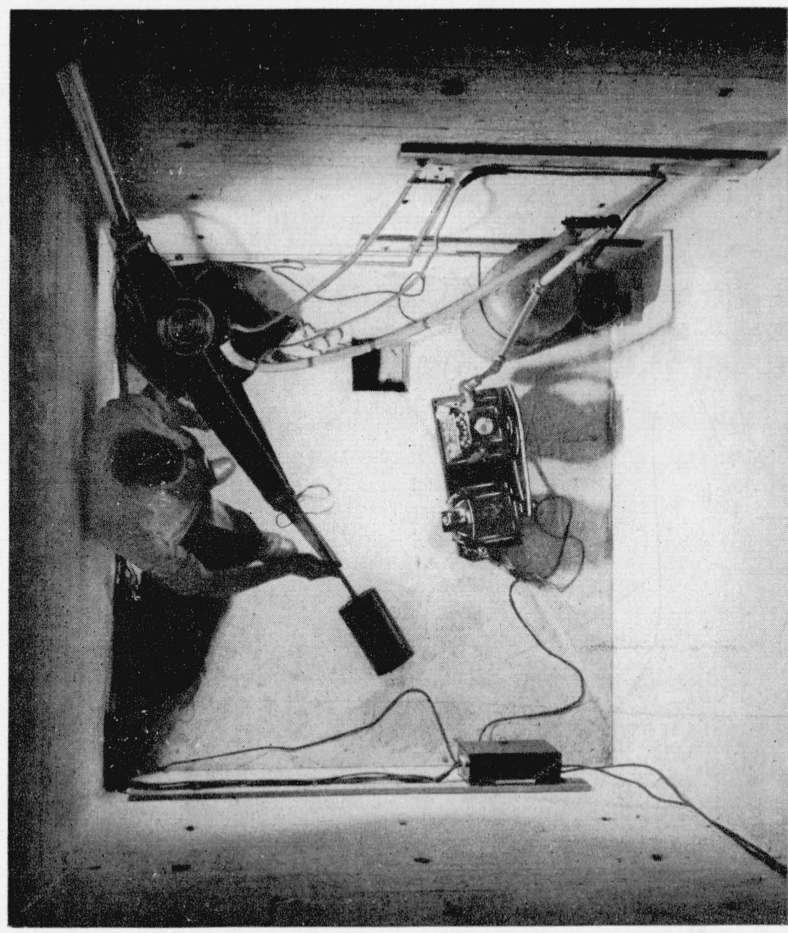

Figure 2. Pit in which the measurements were made.

stant to 3 percent. A good share of this fluctuation in output could be attributed to random focal spot motion. It was not considered excessive for the present purposes.

For narrow-beam conditions, the absorbers were placed directly below the shield diaphragm. The chamber-to absorber distance could thus be varied from approximately 10 to $16 \mathrm{ft}$. The irradiated area of the samples was not more than 3 in. in diameter.

In testing for broad-beam conditions, the absorbers were laid directly over the top of the pit with an overlap of approximately $1 \mathrm{ft}$ all around the lip. The concrete samples were in one piece, either $8 \mathrm{ft}$ by $8 \mathrm{ft}$ by 6 in. or $8 \mathrm{ft}$ by $8 \mathrm{ft}$ by $3 \mathrm{in}$. Lead samples were made up of $2 \mathrm{ft}$ by $8 \mathrm{ft}$ by $1 / 8$ in. thick strips. Parallel strips covered the whole surface of the pit aperture in $1 / 8$ in. thick layers. An overlap of at least $1 / 4 \mathrm{in}$. was provided at each joint. Lap joints were staggered in adjacent layers, but none of the joints came closer than $9 \mathrm{in}$. to a line through the chamber and target. This lead, being quite flexible, required additional support. The pit aperture was reduced by placing plyboard on the basement floor extending over the pit. An unsupported area of lead $3 \mathrm{ft}$ square was obtained in the center of the aperture. (This area 
was found to be adequate for broad-beam conditions with concrete. Smaller dimensions were expected and obtained for lead.)

The large concrete blocks were each weighed to an accuracy of $10 \mathrm{lb}$. Corrections were made for known air holes and reinforcing iron around the unexposed edges. Thicknesses of each slab, measured at a number of distributed points, were found to deviate by no more than $1 / 8$ in. from their mean value. This mean thickness, together with the length, the width, and the corrected weight served to determine the average density. The mean of five additional thickness measurements obtained in the vicinity of the X-ray irradiated area was then corrected to correspond to a specific gravity of $147 \mathrm{lb} / \mathrm{ft}^{3}(2.35 \mathrm{~g} / \mathrm{cc})$. The narrowbeam concrete blocks are those previously used [10.] The accuracy of the concrete dimension measurements is estimated to be within 2 percent.

Approximately 300 measurements of thickness of the lead strips indicated maximum deviation from the mean of the order of \pm 4 percent.

All attenuation curves were obtained for a 7.5-in. chamber-to-barrier distance. This distance was a practical lower limit for our equipment. However, personnel are usually not located closer than 7.5 in. to the protective barrier in most medical X-ray installations. The target-to-chamber distance was thus $175 \frac{1}{2} \mathrm{in}$.

\section{Results}

In order to properly evaluate this experiment, it was first necessary to investigate the extraneous scattering. Evidence of the lack of scattering without absorber may be obtained by observing the dosage rate at different distances from the target. The inverse square law may be used to reduce these dosage rates to a common distance. The variation of the values, so computed, is a measure of the amount of scattering present. Figure 7, A indicates the magnitude of the scattering. It is seen that, with no absorber in place and for the two smaller diaphragms, dosage rates computed back to a fixed distance $(1 \mathrm{~m})$ by the inverse square law remain essentially constant for large variations of the target-to-chamber distance. With the largest diaphragm, the main beam actually begins to strike the side walls about half way down the pit. The resultant scattering from the walls may contribute to the dosage rate measured in the center of the beam. Since the scattering is most important in the forward direction, some increase in the measured radiation should be obtained for chamber positions below the mid-depth of the pit. The experimental evidence verifies this qualitative prediction. Figure $7, \mathrm{~A}$ indicates that the contribution of scattering is about 5 percent of the main beam at a distance of $80 \mathrm{in}$. below the lip of the pit.

Scattered radiation will, of course, be measured with an absorber over the pit. The lack of extraneous scattering from the pit walls may be determined by measuring the radiation very near to the wall. If the radiation measured near to the wall is a small part of that in the center of the beam, then any contribution received at the center of the beam from the wall may be negligible. In figure 3 the pit walls correspond to angles of approximately $-5^{\circ}$ and $95^{\circ}$. The experimental results indicate that the dosage rate is rapidly decreasing as the walls are approached. The amount of radiation scattered from the nearer wall to the center of the beam is thus negligible. The dosage rate measured at the center of the beam and $7.5 \mathrm{in}$. from the absorber therefore does not include an appreciable amount of scattered radiation.

Figure 3 also indicates other interesting items. The sloped central portion of the azimuth curve without absorber has been discussed previously in the literature [9]. It may be attributed to change of the inherent filtration with angle. The asymmetry results from having the target

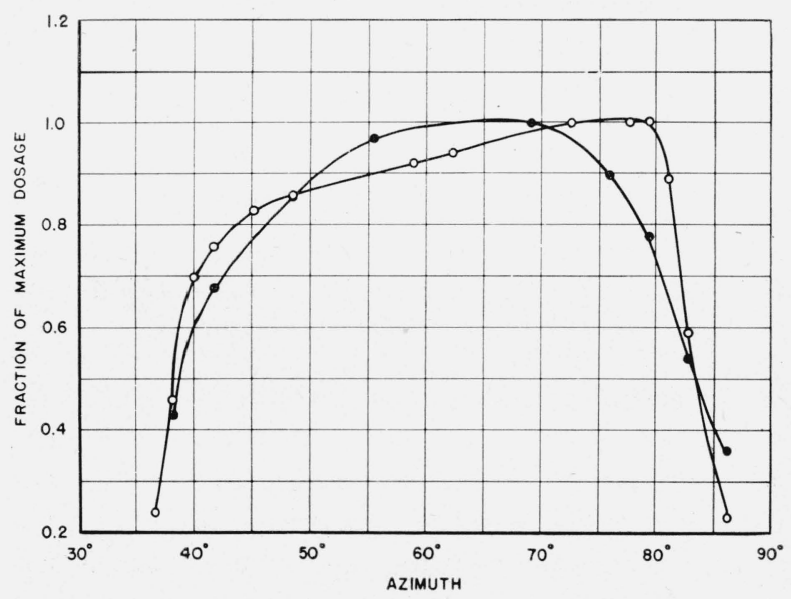

Figure 3. Variation of dosage rate across the X-ray beam for an irradiated area approximately $37 \mathrm{in}$. in diameter.

Open circles were obtained with no barrier. Filled circles were obtained with a 6-in.-thick concrete absorber in place. 
set at $45^{\circ}$ to the emergent beam. Large azimuth angles on this figure correspond to least inherent filtration. The figure also indicates that the presence of an absorber tends to sharpen the main peak but to increase the dosage rate near the foot of the curves. The first factor may be attributed to diminution of the beam by larger thicknesses of material at points different from the normal to the absorber. The second factor may be attributed to the radiation scattered out of the confines of the original beam.

It was found that each diaphragm gave a different apparent tube output. This increase in dosage rate with diaphragm size was attributed to spread of the focal spot. In order to show more clearly the effect of beam size on the attenuation curves, all dosage rates for a given diaphragm and kilovoltage have been reduced by the ratio of the apparent tube outputs - with zero absorberfor that disphragm and for the smallest diaphragm. All four sets of curves for a given voltage thus pass through the same zero absorber point.

Figure 4 shows the experimental attenuation curves for lead. The lower curve at each potential is for narrow-beam conditions. There was no important change in the data for irradiated areas greater than $13 \mathrm{in}$. in diameter. The actual deviation of the dosage rate for variation of the diaphragm was of the same order as the experi-

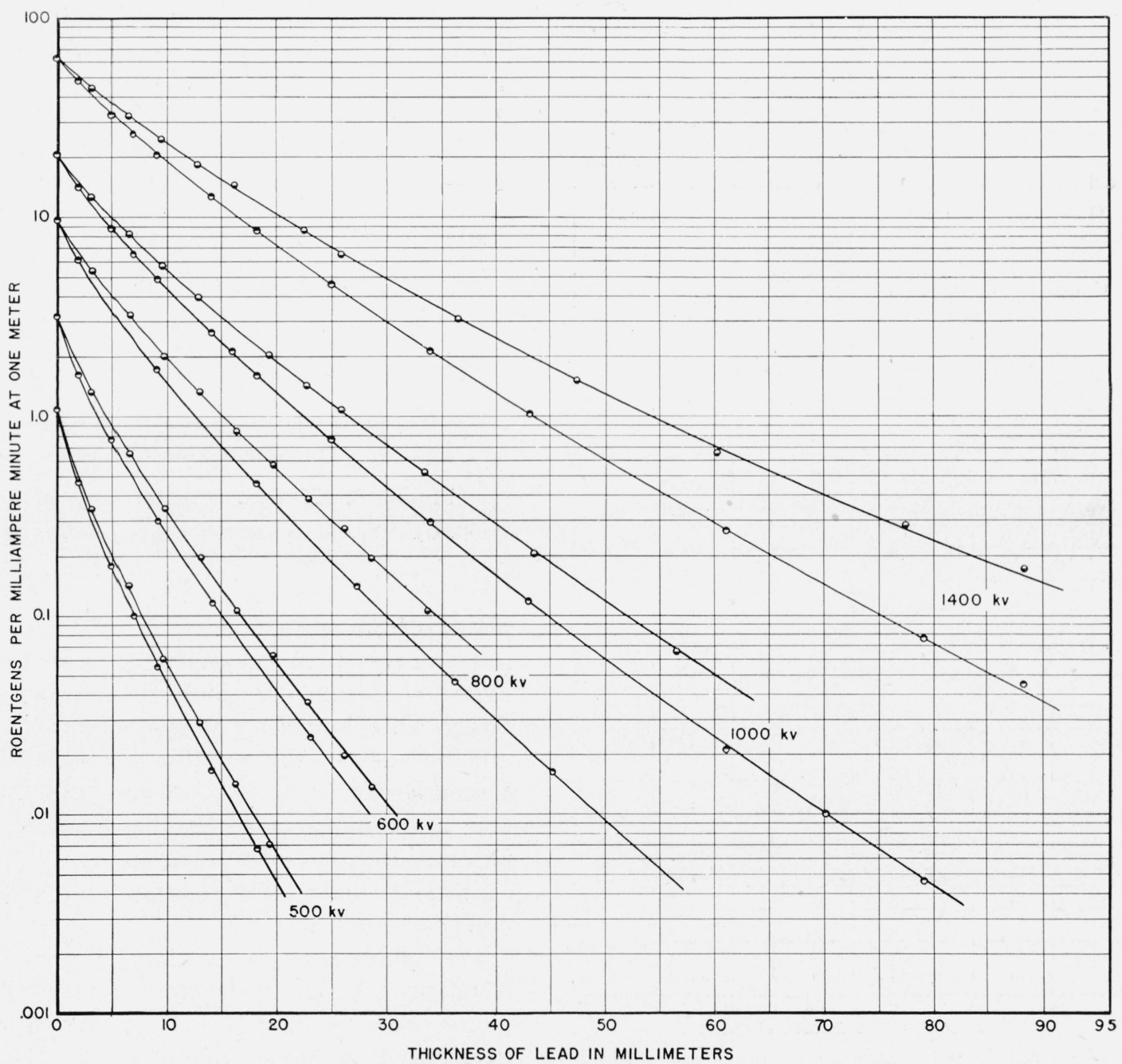

Figure 4. X-ray attenuation curves in lead.

Dosage rates for the three diaphragms under broad-beam conditions were so nearly the same that they could not be separated on this scale. beams (diameter, 13 in.); $\Theta$, narrow beam. 
mental error. There is, however, an interesting change in the difference between narrow- and broad-beam absorption curves with potential producing the X-rays. In agreement with the qualitative predictions this difference becomes more important for large photon energies, since the scattered photons are not so readily absorbed photoelectrically.

Figures 5 and 6 show the attenuation curves for concrete. For each generator potential the lowest curve is for narrow-beam conditions, that is, when a negligible amount of the dosage is due to scattering from the absorber. The difference between successive curves with narrow-beam, 13, 26 , and 37 in., irradiated diameter indicates the magnitude of the scattering from the corresponding ring of barrier. It is evident that the scattered

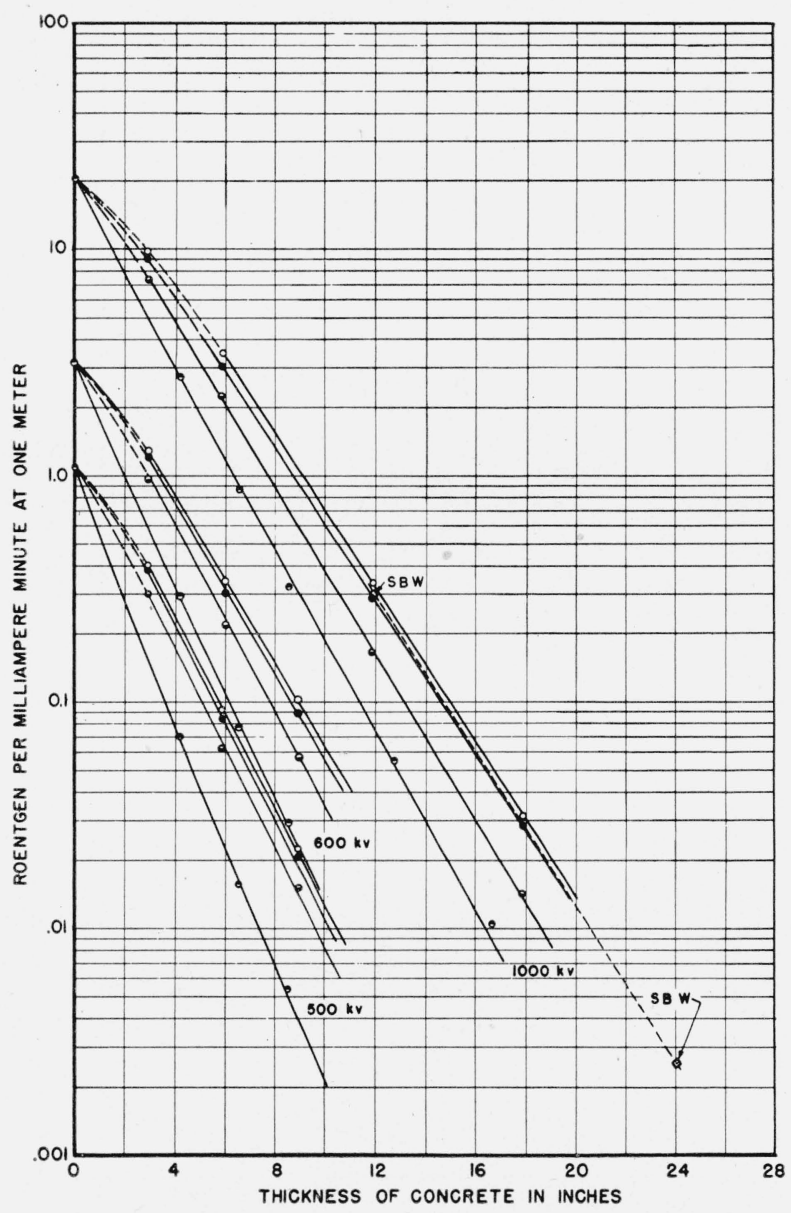

Figure 5. X-ray attenuation curves in concrete.

The density of the concrete is $147 \mathrm{lb} / \mathrm{ft}^{3}$. The dotted line is drawn through two experimental points obtained from published work of Singer, Braestrup, and Wyckoff with a resonance-type generator. $\odot$, Narrow beam; $\bullet, 13$-in. beam; , 26-in. beam; $\bigcirc, 37$-in. beam.

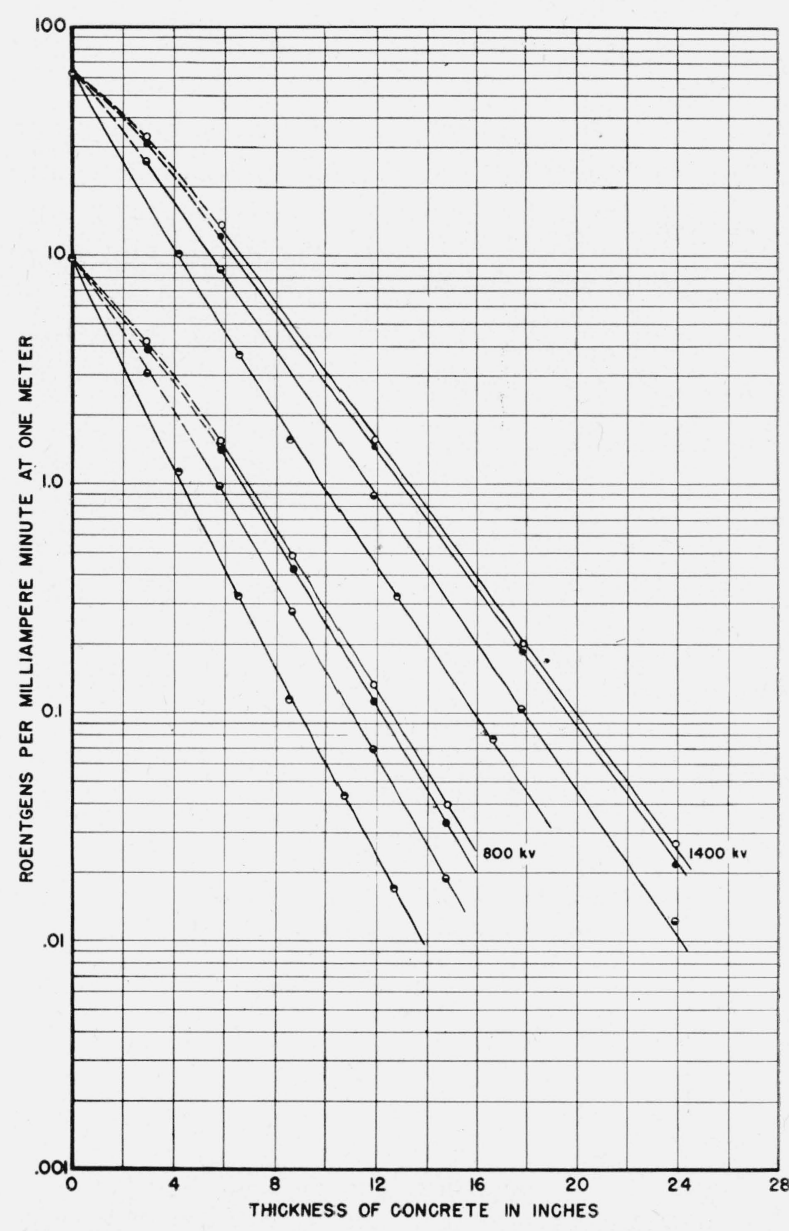

Figure 6. X-ray attenuation curves in concrete.

The density of the concrete is $147 \mathrm{lb} / \mathrm{ft}^{3}$. $\odot$, Narrow beam; $\odot, 13$-in. beam; , 26-in. beam; $\bigcirc, 37$-in. beam.

photons from the successive outer rings contribute less and less to the dosage rate measured on the axis of the beam. Because of the small change in dosage rate between the 26 -in. and $37-$ in. beam and because of the rapidly decreasing contribution of scattering with beam size, the 37 -in. diameter beam in concrete has been assumed for all practical purposes to correspond to broad-beam conditions under the experimental arrangement described above.

For comparison with data obtained from a resonance X-ray generator, two experimental points from Singer, Braestrup, and Wyckoff [1] have been included on figure 6 . These were obtained with a two-million-volt resonance type unit operating at one million volts and giving an irradiated area of approximately $4 \frac{1}{2} \mathrm{ft}$ by $7 \mathrm{ft}$. 
The target-to-chamber distance was approximately 8 and $9 \mathrm{ft}$. These data have been corrected as nearly as possible to the experimental conditions used for our new data. The effect of the wave form on the X-ray output and the effect of the shorter target-barrier distance on the amount of the scattering measured in protection studies cannot be rigorously determined with our present knowledge. One may say, however, that the target current flows appreciably only when the alternating voltage peak is above $800 \mathrm{kv}$. The effective potential should therefore be somewhere between 800 and $1,000 \mathrm{kv}$. The slope of an attenuation curve is often taken as a measure of the effective potential of the X-rays. The points [1] give a line whose slope is midway between that here reported for 800 and $1,000 \mathrm{kv}$. The results are thus in qualitative agreement.

There is evidence, in the lead as well as the concrete curves, of a reduction in the slope of the attenuation curve with irradiated area. This effect is especially pronounced in the change from narrow beam to 13 -in. diameter beam. There is also evidence in concrete, but not in lead, of the low energy scattered radiation. Both of these items tend to increase the barrier thickness requirements for broad beam over those for narrow beam.

Figure 7 for data taken at $1,000 \mathrm{kv}$, shows the importance of barrier-to-chamber distances. These curves were obtained by varying the elevation of the chamber in the pit. Figure $7, \mathrm{~B}$ is for a lead absorber placed over the pit. The three diaphragms all give essentially the same dosage rate at a distance of 7.5 in. below the absorber. Broadbeam conditions were therefore obtained at this position for an irradiated area of 13-in. diameter. As the barrier-to-chamber distance is increased, different diaphragms do not give the same dosage. Broad-beam conditions, therefore, do not exist in this intermediate region for the diaphragms used. At very large distances, the dosage rates appear to become constant. This condition corresponds to the requirements for narrow beam. As would be expected, the distance from the barrier for narrow-beam conditions increases with the size of the irradiated area. After correction for scattering, the dosage rate of the largest beam also agrees with that of the other beams at a distance of 78 in. Moreover, this constant dosage rate agrees with

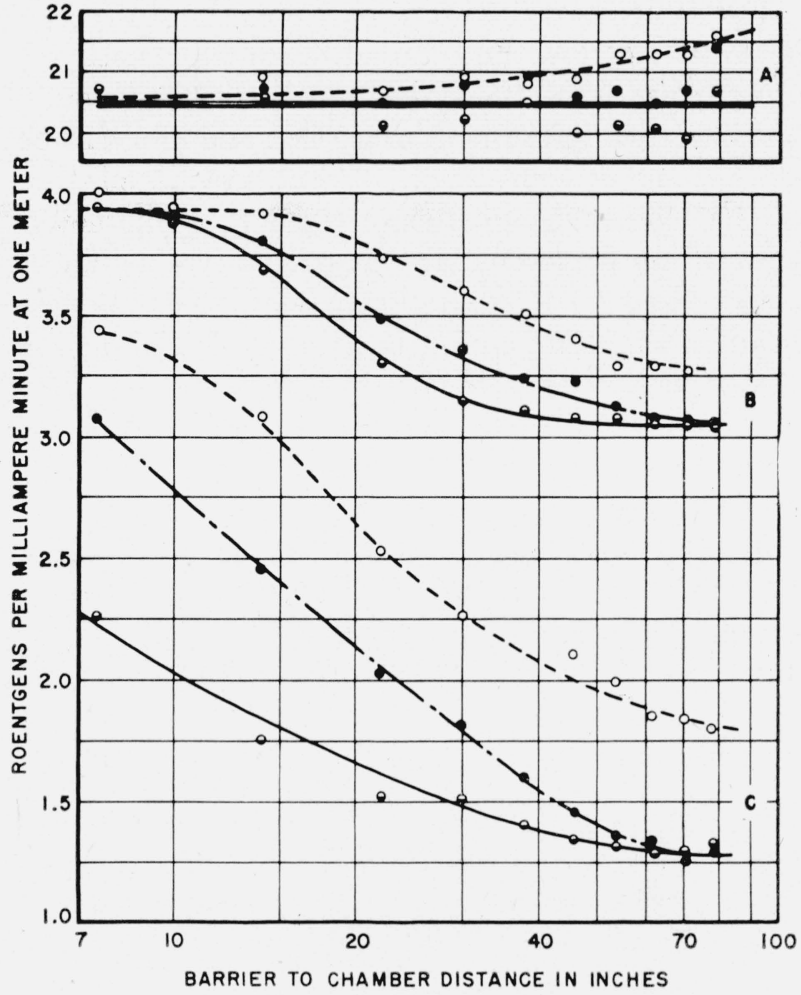

Figure 7. Variation of X-ray dosage with distance from the lip of the pit for 1,000 kv.

A, With no absorber in the beam; B, with $12.9 \mathrm{~mm}$ of lead over the pit; C, with $5.8 \mathrm{in.} \mathrm{of} \mathrm{concrete} \mathrm{over} \mathrm{the} \mathrm{pit.} \ominus, 13$-in. beam;, 26 -in. beam: $\bigcirc$ 37-in. beam.

that obtained for the narrow-beam curves of figure 4. For the concrete sample indicated (fig. 7, C) it appears that the two smaller diaphragms approach narrow-beam conditions for a chamberto-barrier distance of the order of 55 to 78 in. The dosage rate is here constant (1.3) and in agreement with the narrow-beam curve of figure 5 , which also gives a value of 1.3 roentgens per milliampere minute at $1 \mathrm{~m}$.

\section{Conclusions}

Because of the small irradiated areas required for broad-beam conditions in lead, the broadbeam attenuation curves should be used for nearly all cases where lead barriers are considered in this voltage range. The extra thickness of lead required for broad-beam conditions over that for narrow beam varies rapidly with X-ray tube potential. This extra thickness requirement varies from approximately 10 percent at $500 \mathrm{kv}$ to 25 percent at $1,400 \mathrm{kv}$ 
For concrete protective barriers, the difference between narrow- and broad-beam thickness requirements is of the order of $1 \frac{1 / 2}{2}$ to 2 half-value layers, depending upon the portion and kilovoltage of the curves considered. If the irradiated area of the barrier is not more than $3 \mathrm{ft}$ in diameter, and if all personnel are restricted to distances larger than $10 \mathrm{ft}$ from the barrier, then narrowbeam attenuation curves may be used in designing $\mathrm{X}$-ray protection. Such restrictions are not usually feasible, however, because of space limitations. In addition, the cost of requiring the extra space may be larger than the saving in barrier cost.

For the majority of X-ray installation designs, therefore, the broad-beam attenuation curves should be used.

\section{References}

[1] G. Singer, C. B. Braestrup, and H. O. Wyckoff, J. Research NBS 37, 147 (1946) RP1735.

[2] F. Heidenreich and R. Jaeger, Physik. Z. 39, 541 (1938).

[3] R. Jaeger and A. Trost, Elektro. Z. 61, 1025 (1940).

[4] G. W. C. Kaye, W. Binks, and G. E. Bell, Brit. J. Radiol. 11, 676 (1938)

[5] G. C. Laurence, L. W. Ball, and W. J. Archibald, National Research Council of Canada (1942).

[6] T. R. Folsom and E. F. Focht, Am. J. Roentgenology Radium Therapy 5\%, 76 (1944).

[7] E. E. Charlton and H. S. Hubbard, Gen. Elec. Rev. 43, 272 (1940).

[8] G. Failla, Am. J. Roentgenology Radium Therapy 54, 553 (1945).

[9] M. C. Reinhard and H. L. Goltz, Radiology 37, 335 (1941).

[10] G. Singer, L. S. Taylor, and A. L. Charlton, Radiology 33, 68. (1939).

Washington, May 25, 1948. 